\title{
BLENDED LEARNING. POSIBILIDADES DE APLICACIÓN EN LA UNIVERSIDAD TECNOLÓGICA DE CANCÚN
}

\section{BLENDED LEARNING: POSSIBILITIES APPLICATION IN THE TECHNOLOGICAL UNIVERSITY OF CANCUN}

\author{
Antonio Pérez De la Cruz* \\ Profesor de la División Económico-Administrativas de la \\ Universidad Tecnológica de Cancún \\ [Recepción: Febrero de 2011/ Conformidad: Abril de 2011]
}

\section{RESUMEN}

En la actualidad, las competencias relacionadas con las Tecnologías de Información y Comunicaciones (TIC's) cada día adquieren mayor importancia por contribuir a la creación de valor de las empresas, principalmente en el área de formación del capital humano, en la cual las tendencias apuntan hacia las modalidades de enseñanza aprendizaje en línea. Por ello, las universidades deben comenzar a implementar modelos de enseñanza aprendizaje en línea, combinadas con las clases presenciales, con la finalidad de que los estudiantes adquieran experiencia en el uso de las TIC's en su formación. Por lo anterior, el presente trabajo de investigación pretende proporcionar un acercamiento al modelo b-larning, explicar que elementos lo componen y por qué este modelo está más acorde al modelo por competencias profesionales, el rol del docente y el estudiante en el modelo b-learning, así como conocer las posibilidades de implementación del blearning, identificando inicialmente si los docentes y estudiantes cuentan con los recursos, medios, conocimientos e interés en el uso de las TIC's en los procesos de enseñanza aprendizaje, a través de un estudio realizado a docentes y alumnos de la División Económico Administrativas de la Universi-

\begin{abstract}
At present the present time the skill related to the Technologies of Information and Communication (TIC's) acquiere every day more importance in the contribution to business success, mainly in the area of formation of human capital, in which in-line education trends. For this reason, the universities must begin to implement education model in-line learning, combined with the actual clases, with the objective that the students acquire experience in the use of the TIC's. In consecuence, the present investigaction provides an approach to the model of b-learning to explain what elements are included and the studen and teacher's roll, as well as to Know the possibilities for its implementation and identifying, initially, if we count with the necessary resources, means, knowledge and interest in the use of the TIC's in the processes of an already realised study of eduction and student of Business Administration of the Divisiñon Económico administrativa of the Universidad Tecnológica de Cancún. And finally to reach conclusions and recommendations for its application.
\end{abstract}

\footnotetext{
* Profesor de la División Económico-Administrativas de la Universidad Tecnológica de Cancún - México
} E-mail: aperez@utcancun.edu.mx 
dad Tecnológica de Cancún, para finalmente llegar a conclusiones y recomendaciones de aplicación.

Palabras Claves: Formación de capital humano, Aprendizaje combinado, Tecnologías de la Información y Comunicaciones.
Key words: Training human capital, Blended Learning, Information Technologies and Communications.

\section{INTRODUCCIÓN}

Las Tecnologías de la Información y Comunicaciones (TIC's), cada día adquieren mayor importancia como fuente de creación de valor en las organizaciones, entre otras cosas porque han influido, tanto en la forma en que se relacionan, se comunican y trabajan los individuos, como en la forma en que se gestionan los procesos de producción, distribución, promoción y comercialización de los productos y servicios.

En este sentido, en la actualidad ningún sector de la economía puede ignorar o menospreciar dos cosas respecto al desarrollo y evolución de las TIC's, por un lado, que los avances en las TIC's han creado nuevos escenarios y una reorganización de roles de los individuos, empresas e instituciones gubernamentales, y por otro, que estos mismos avances proporcionan diversas alternativas de solución a las nuevas problemáticas de la sociedad.

En este escenario es evidente que los avances tecnológicos y sus efectos en la sociedad, requiere que los individuos posean un mayor conocimiento y dominio de herramientas y medios relacionados con las TIC's, que le permitan desempeñarse eficientemente en sus puestos de trabajo actuales y futuros. Así mismo, es un hecho que en la actualidad las empresas que quieren crear, mantener o mejorar sus niveles de competitividad, están canalizando sus esfuerzos a mejorar sus sistemas y modelos de negocio haciendo uso de nuevas tecnologías, como lo es la intranet de la empresa, el Messenger, el skype y los dispositivos móviles como PDA, smartphones, Iphones y BlackBerry con la finalidad de lograr una mayor, mejor y más rápida comunicación entre empleados y directivos.

Por otro lado, las tecnologías también contribuyen a proporcionar un valor agregado a los clientes al poner a su disposición más canales de comunicación y comercialización con la empresa, como lo es un sitio web dinámico que permita transacciones de comercio electrónico y cuentas de la empresa en redes sociales como Facebook, Twitter o Youtube que permiten recibir opiniones, comentarios o quejas de los clientes, así como la promoción de los productos y servicios de la empresa.

En este orden de ideas, un rubro que se ha visto influenciado por las TIC's en el sector empresarial y que no puede pasar desapercibido, es el área de Recursos Humanos, específicamente en la formación de personal, en donde cada vez cobra más fuerza las modalidades de formación a distancia a través de Internet y de dispositivos móviles, que se complementan con la formación presencial que se imparte en la empresa, permitiendo aprovechar las ventajas de cada modelo.

Tomando en cuenta lo anterior, es determinante que el sector educativo adapte sus sistemas, procesos, carreras, programas y modelos a las demandas del sector produc- 
tivo, y comience a incluir en un plan estratégico, medios tecnológicos en sus procesos de enseñanza aprendizaje, principalmente en esta modalidad combinada que cada día cobra más fuerza en el sector productivo, es decir, usando plataformas educativas en línea que se usen en forma paralela con las clases presenciales, de tal forma que permitan a los alumnos, por un lado, estudiar de forma anticipada temas que se verán en clase, reforzar conocimientos y desarrollar habilidades, actitudes y valores, tanto en forma presencial como a distancia, y por otro, que en el momento de incorporarse al mercado laboral, se les facilite adaptarse a los modelos de formación que se están usando en las empresas.

En este orden de ideas, cuando se pretende implementar en una institución educativa, modelos de enseñanza aprendizaje que impliquen el uso de las TIC's algunas de las preguntas que surgen en relación a los docentes y los alumnos son:

a. ¿Poseen los recursos y medios tecnológicos necesarios para llevar a cabo una iniciativa de este tipo?

b. ¿Cuentan con los conocimientos y habilidades mínimas para usar la tecnología requerida?

c. ¿Tienen interés? o dicho de otro modo, ¿existe una demanda real de un servicio de este tipo?

d. Y finalmente, si cuentan con los medios tecnológicos zqué uso le dan?, ¿cómo y dónde los usan?, ipueden usar estas tecnologías en sus procesos de enseñanza aprendizaje?

Por lo anterior, y con la finalidad de dar respuesta a estas interrogantes, en este trabajo se muestran los resultados de un estudio de investigación realizado en mayo-agosto del 2009, como parte de los trabajos realizados por el Cuerpo Académico de Desarrollo de Capital Humano de la División Económi- co Administrativas de la Universidad Tecnológica de Cancún.

\section{OBJETIVO GENERAL}

Conocer la viabilidad de implementación del modelo Blended Learning en los procesos de enseñanza aprendizaje en la División Económico-Administrativas de la Universidad Tecnológica de Cancún.

\section{OBJETIVOS ESPECÍFICOS}

- Medir el porcentaje de estudiantes y docentes de la División Económico-Administrativas de la Universidad Tecnológica de Cancún que tienen acceso a las TIC's y el nivel de conocimiento que poseen en el uso y las características de estas tecnologías.

- Descubrir si los estudiantes y docentes de la División Económico-Administrativas de la Universidad Tecnológica de Cancún tienen interés en el uso de las TIC y consideran que son capaces de usarlas en sus procesos de enseñanza aprendizaje.

- Identificar los medios y dispositivos tecnológicos con los que cuentan los estudiantes y docentes de la División Económico-Administrativas de la Universidad Tecnológica de Cancún.

\section{MARCO CONCEPTUAL}

\section{Un acercamiento al modelo B-learning}

El Blended Learning (B-learning) es un aprendizaje combinado, híbrido o mixto del modelo presencial y en línea (Garcia, 2009). Una definición más amplia es la que proporciona González (2005), quién lo describe de la siguiente manera: "es un combinado entre la formación a distancia on-line y la formación tradicional, de manera que aúna las ventajas de ambos sistemas de formación”. 
Al llevar a cabo el proceso de enseñanza aprendizaje en forma combinada, el b-learning amplía las posibilidades de los docentes y estudiantes al dotarlos de mayor cantidad de recursos y medios, que permiten eficientar el proceso. Además de lo anterior, contribuye a que los estudiantes desarrollen competencias en el área de las TIC, que como se menciono anteriormente, en la actualidad son indispensables en el sector productivo, principalmente porque el desarrollo del capital humano, cada vez más frecuentemente incluye la formación a través de las TIC, entre otras cosas, por los beneficios en costos y resultados que les proporciona a las organizaciones.

El concepto de las TIC aplicadas a la educación, cada vez es más incluyente, en cuestión de recursos, medios y modelos teóricos. Hoy en día, las herramientas tecnológicas que pueden aplicarse en la educación incluyen Internet, las redes sociales y los dispositivos móviles como PDA, Palm, smartphones, reproductores de $\mathrm{Mp} 3$ y Mp4.

Por otro lado, los modelos teóricos que predominan, tanto en el sector educativo como empresarial, en materia de formación, están orientados al modelo por competencias, tanto laborales como profesionales, y aunque existen diferencias sustanciales entre dichos modelos, la formación en competencias en ambos sectores tienen algunos puntos en común, por un lado, en ambos sectores se ha llegado a la conclusión de que las competencias tienen que ser observadas para medir el desempeño, y por otro, que lo consideran como algo integrado que incluye el saber, saber hacer y saber ser, aunque en el sector productivo se agregan: el saber estar, el querer hacer y el poder hacer, los cuales no se contemplan en el sector educativo.

En este sentido, la combinación del modelo por competencias y el b-learning, ofrece mayores ventajas que la formación e-learning o la presencial, ya que como se menciono, permite aprovechar las ventajas de ambos modelos.

\section{Elementos que componen el B-learning}

El b-learning incluye tres elementos fundamentales:

\section{MODELO BLENDED LEARNING}

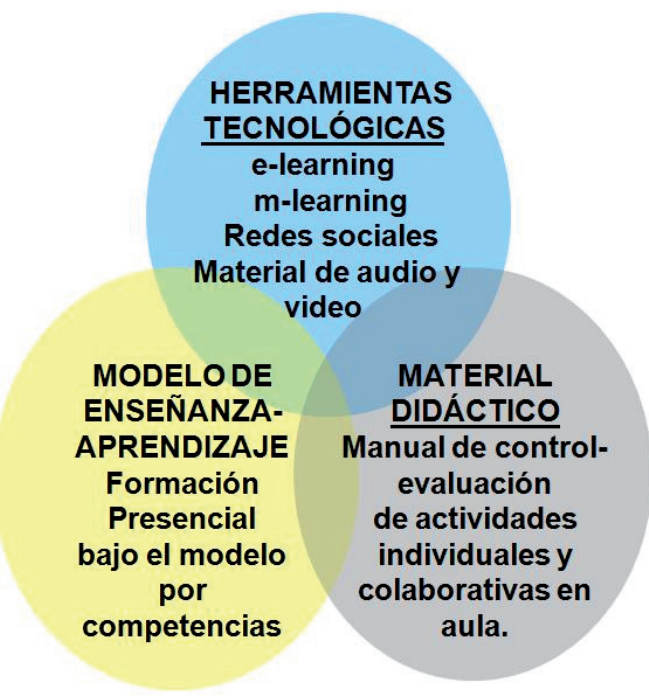

1. Un Modelo de enseñanza aprendizaje, que en el caso de la Universidad Tecnológica de Cancún es el Modelo por Competencias Profesionales.

2. Las herramientas tecnológicas a utilizar, que incluyen el e-learning, el m-learning, las redes sociales y material de audio y video. En el caso de la Universidad, la plataforma que se contempla utilizar es el administrador de contenidos educativos en línea Moodle.

3. El Material didáctico, la clave del modelo consiste en cambiar la forma en que se imparte la clase presencial y para ello se requiere de elaborar y proporcionarle a los alumnos un Manual que incluya, además de los contenidos, las actividades individuales y colaborativas que llevará a 
cabo en la clase presencial, la calendarización de las actividades y los sistemas de control y evaluación del desempeño.

\section{¿Por qué el b-learning es un modelo más acorde con el modelo por competencias profesionales?}

De acuerdo a Pereda y Berrocal (2009, p. 76), las competencias se definen como: "un conjunto de comportamientos observables que están causalmente relacionados con un desempeño bueno o excelente en un trabajo concreto y una organización concreta”. Estos comportamientos solo pueden evaluarse a través de la observación del desempeño.

En este marco, es importante señalar que independientemente del sector de que se hable, ya sea educativo o empresarial, las competencias se evidencian mediante la observación de la forma en que se aplican los saberes, las habilidades y actitudes al llevar a cabo una actividad concreta, lo cuál otros modelos que hacen uso de las TIC's, como el e-learning o el m-learning no lo permiten.

En este sentido y considerando que no se puede desligar el contexto socioeconómico, el sector empresarial y el sector educativo, es importante tener en cuenta los siguientes puntos, en la realidad actual:

1. "El vertiginoso avance de la tecnología especialmente de la informática, ha generado otro tipo de mentalidad y de habilidades en los estudiantes" (Espíndola, 2000, p. 268).

2. Desde el punto de vista de la educación Gónzalez y Wagenaar (2003, citado en Bautista, Borger y Forés, 2006) comentan lo siguiente:

En el eje de la formación actual, las nuevas propuestas universitarias abogan porqué el estudiante sea el centro y protagonista del proceso de aprendizaje, y se sustituya a la importancia de la enseñanza y la adquisición de conocimientos por la importancia del aprendizaje y la adquisición de competencias (p. 20).

3. En los entornos virtuales de enseñanza aprendizaje, Zulma (2006) señala que se ponen de manifiesto los siguientes aspectos:

Los alumnos planifican sus acciones, se fijan metas, se organizan, se observan a sí mismos y toman decisiones sobre sus acciones futuras. En relación con lo motivacional, son estudiantes con alta autosuficiencia, se sienten responsables de sus aprendizajes y suelen tener interés intrínseco en las tareas. Por ello, es común que desplieguen esfuerzos importantes y persistan al realizar las actividades. Como rasgos de comportamiento, los estudiantes autorregulados seleccionan, estudian y crean ambientes que optimizan el aprendizaje. Tratan de buscar consejo, información y lugares donde es más factible el aprendizaje (p. 46).

$\mathrm{Si}$ a este último punto se añade estrategias docentes en modalidad presencial, que como sugiere Ferreiro (2009), partan de un tipo de relación diferente maestro-alumno, como son: la mediación y la cooperación, el proceso de enseñanza aprendizaje se optimiza. De acuerdo al autor el mediador es la persona que al relacionarse con otros:

- Favorece su aprendizaje.

- Estimula el desarrollo de sus potencialidades.

- Y, lo más importante, corrige funciones cognoscitivas deficientes.

El otro tipo de relación que Ferreiro (2009) señala como estrategia en el aula, es la cooperación, la cual aporta, entre otras cosas:

- Modelos que pueden imitar.

- Oportunidades de hacer, decir y sentir. 
- Apoyo según las necesidades manifiestas.

- Expectativas in crescendo.

- Autorregulación personal y en equipo.

- Reforzamiento positivo constante.

- Perspectivas diferentes sobre un mismo asunto.

- Desarrollo de las habilidades cognitivas, pero también sociales y afectivas.

Tomando en cuenta lo anterior, la propuesta del b-learning supone, que la combinación de medios y recursos presenciales y en línea, proporcionará a los estudiantes y a los docentes mayores ventajas que las que ofrecen cada modelo por separado, lo cual cobra relevancia, cuando el sector en el que se desenvolverán los estudiantes, esta caracterizado por constantes cambios y orientado a la aplicación de las competencias de manera eficaz y eficiente, con el fin de responder de manera oportuna y anticipada a la dinámica de los mercados.

En este sentido, el modelo b-learning, contribuye de manera significativa a desarrollar una de las competencias más importantes de los individuos en el entorno socioeconómico actual, y que desde el informe de Jaques Delors presentado por la UNESCO en 1996 cambio la concepción de la educación, es decir, la competencia de aprender a aprender.

\section{Rol del docente y el estudiante en el mo- delo b-learning}

Tomando en cuenta que el b-learning involucra un nuevo modelo de enseñanza aprendizaje, los docentes y los estudiantes no pueden, como señalaba Albert Einstein en una de sus citas más famosas, "esperar resultados distintos cuando se siguen haciendo las mismas cosas", o bien trasladar los mismos errores del modelo presencial a una plataforma tecnológica, lo cual Cabero (2006) expresa de la siguiente manera:
Un error que siempre hemos cometido con las nuevas tecnologías, y que ha llevado a que las mismas no desarrollen todas las posibilidades que presentan para la creación de nuevos entornos formativos, es el deseo de trasladar sobre ellas principios aplicados, de la enseñanza presencial o de tecnologías más tradicionales.

De acuerdo a Cabero (2007) algunos de los roles del docente cuando se implementan las TIC en los procesos de enseñanza aprendizaje son los siguientes:

- Facilitador del aprendizaje: El docente de esta forma pasa de ser un experto en contenidos y en su transmisión verbal a un facilitador el aprendizaje, lo cual le va a suponer que realice diferentes cuestiones como son: diseñar experiencias de aprendizajes para los estudiantes, ofrecer una estructura inicial para que los alumnos comiencen a interaccionar, animar a los estudiantes hacia el autoestudio, o diseñar diferentes perspectivas sobre un mismo tópico.

- Trabajar en equipo y en colaboración con otros profesionales: el profesor va a jugar un papel importante en el diseño de medios, materiales y recursos adaptados a las características de sus estudiantes, materiales que no sólo serán elaborados por él de forma independiente, sino en colaboración, tanto con el resto de compañeros involucrados en el proceso, como con otra serie de expertos.

- Poseer una formación y capacitación técnica e instrumental: el docente debe saber interaccionar con las TIC, tener una posición significativa hacia las mismas como herramientas de comunicación e interacción con la información, saber interaccionar, evaluar y seleccionar la información que se nos ofrece por las nuevas tecnologías, y utilizar los medios como 
instrumentos de expresión y creación de mensajes.

En cuanto a los roles de los estudiantes, Bautista et al. (2006, citado en Borges, 2007) menciona lo que se puede esperar de ellos, en entornos virtuales de aprendizaje:

- Implicación personal y responsabilidad en su desempeño como estudiante.

- Respeto a los compañeros y a sus opiniones y propuestas.

- Leer y escribir reflexivamente, con criterio propio.

- Que actúen honestamente, que no copien trabajos de otros o de otras fuentes y los hagan pasar por propios.

- Que pregunten, que participen, que aporten en el aula virtual.

- Que sepan qué canales de ayuda existen y que los utilicen si necesitan ayuda o aclaraciones.

- Que estén dispuestos «a explorar, a experimentar y a aprender de otra manera».

Por su parte, Pacheco (2007) menciona que existen aspectos que el estudiante inicialmente presencial debe adaptar en el proceso de virtualizar su aprendizaje, que van desde la planificación de su aprendizaje menos dirigida por el profesor, la organización apropiada de su tiempo, interacción social virtual, la cual tiene características diferentes de la presencial y un desarrollo diferente de actividades de aprendizaje.

Tomando en cuenta lo anterior, Borges (2007, citado en Pacheco, 2007) señala que los buenos estudiantes en entornos virtuales, presentan las siguientes características:

- Creen que el aprendizaje de calidad puede tener lugar en cualquier entorno, sea presencial o a distancia.

- Saben que aprender en un entorno virtual no resulta más fácil necesariamente.

- Relacionan su vida real con lo que aprenden y viceversa.
- Manejan adecuadamente la ambigüedad o la incertidumbre que se puede dar en ocasiones al aprender en un entorno virtual de enseñanza aprendizaje.

- Organizan su tiempo adecuadamente, de forma que compatibilizan su dedicación académica con sus obligaciones laborales y familiares.

- Construyen su propio conocimiento a partir del material de estudio y también de la relación con los compañeros y el profesor: aprenden de sus compañeros y profesor, y aprenden con ellos también.

- Muestran una gran motivación y una gran autodisciplina, y las conservan durante el curso a pesar de las dificultades que puedan encontrar.

- Utilizan, si es necesario, los canales de petición de ayuda que la institución pone a su disposición.

- Ayudan a los compañeros, están dispuestos a colaborar y a mantener una buena atmósfera en el aula virtual.

- Tienen una actitud proactiva y son autónomos en la medida de lo posible; muestran iniciativa en su aprendizaje y en su desempeño durante el curso.

- Se comunican con su profesor si tienen dudas o problemas.

\section{RESULTADOS DE LA ENCUESTA APLICADA A LOS ESTUDIANTES DE LA DIVISIÓN ECONÓMICO-ADMI- NISTRATIVAS DE LA UTCANCUN}

En relación a los estudiantes, se aplico la encuesta al 96\% de la matrícula, 169 hombres y 178 mujeres. En el caso de los estudiantes de TSU el 60\% tenían edades entre los 18 y 20 años y el $40 \%$ restante manifestó tener edades entre 21 y 30 años, con un solo estudiante con edad mayor a 30 años. En la carrera de Ingeniería la mayor parte de 
los encuestados en el momento del estudio, tenían edades entre 21 y 30 años (78\%), el $12 \%$ edad entre 18 y 20 años y el $9 \%$ con edad mayor a 30 años.

\begin{tabular}{|l|l|}
\hline \multicolumn{1}{|c|}{$\begin{array}{c}\text { DOCENTES } \\
\text { (33 encuestados) }\end{array}$} & \multicolumn{1}{c|}{$\begin{array}{c}\text { ESTUDIANTES } \\
\text { (347 encuestados) }\end{array}$} \\
\hline 11 Profesores de & $>$ 210 de la carrera de \\
Tiempo Completo & TSU en Administración y \\
22 Profesores de de & TSU en Contaduría \\
Asignatura & $>\quad 137$ de la carrera de \\
& $\begin{array}{l}\text { Ingeniería en Desarrollo e } \\
\text { Innovación Empresarial. }\end{array}$ \\
\hline
\end{tabular}

La encuesta estaba formada por 24 preguntas, 5 de información general del encuestado y 19 orientadas a conocer: a) la disponibilidad, acceso y uso del medio, b) el conocimiento de las características y aplicaciones del medio y c) la percepción e interés en la implementación del modelo blearning.

\section{DISPONIBILIDAD, ACCESO Y USO A LAS TIC'S}

1. De los siguientes dispositivos ¿cuál o cuáles tienes actualmente?

\begin{tabular}{|l|r|}
\hline \multicolumn{1}{|c|}{ DISPOSITIVO } & RESP \\
\hline Celular o Smartphone & 386 \\
\hline Reproductor de Mp3 y Mp4 & 176 \\
\hline PDA & 13 \\
\hline Ninguno de los anteriores & 7 \\
\hline Otros & 8 \\
\hline
\end{tabular}

Esta pregunta era de opción múltiple por lo que los resultados sobrepasan el $100 \%$.

Llama la atención que solo 7 personas de 347 encuestados, manifestaron no contar con ninguno de estos dispositivos y 8 comentaron tener otro tipo de dispositivo, diferente a los mencionados.
2. ¿Cuál es la actividad que realizas con estos dispositivos?

\begin{tabular}{|l|c|}
\hline USO & RESP \\
\hline Escuchar música & 304 \\
\hline Reproducir videos & 218 \\
\hline Escuchar radio & 118 \\
\hline llamadas telefónicas & 261 \\
\hline Intercambiar archivos de audio & 202 \\
\hline Intercambiar archivos de video & 157 \\
\hline Intercambiar archivos de datos & 173 \\
\hline Acceso a Internet & 185 \\
\hline
\end{tabular}

Esta pregunta era de opción múltiple por lo que los resultados sobrepasan el 100\%.

3. ¿Tienes acceso a internet en tu casa?

\begin{tabular}{|l|c|}
\hline ACCESO A INTERNET & RESPUESTAS \\
\hline SI & 282 \\
\hline No & 65 \\
\hline
\end{tabular}

4. Si no tienes Internet en tu casa ¿Dónde te conectas a Internet?

\begin{tabular}{|l|c|}
\hline \multicolumn{1}{|c|}{ ACCESO A INTERNET } & RESPUESTAS \\
\hline En trabajo & 156 \\
\hline Escuela & 119 \\
\hline En un cibercafé & 72 \\
\hline
\end{tabular}

5. ¿Cuentas con laptop o computadora en tu casa?

\begin{tabular}{|l|c|}
\hline COMPUTADORA EN CASA & RESPUESTAS \\
\hline $\mathrm{Si}$ & 315 \\
\hline No & 32 \\
\hline
\end{tabular}

\section{CONOCIMIENTO DE LAS CARACTE- RÍSTICAS Y APLICACIONES DE LAS TIC'S}

En esta clasificación se plantearon preguntas orientadas a conocer si los estudiantes conocían las características técnicas de sus dispositivos en cuanto a posibilidades de acceso y capacidades de almacenamiento. 
6. ¿Qué capacidad de almacenaje tiene tu dispositivo?

7. ¿Tu dispositivo cuenta con acceso a Redes Inalámbricas?

8. ¿Tu dispositivo cuenta con conexión Bluetooth?

9. ¿Tu dispositivo cuenta con tarjetas de almacenamiento adicionales a la memoria interna del equipo?

\begin{tabular}{|l|c|}
\hline \multicolumn{1}{|c|}{$\begin{array}{c}\text { CONOCIMIENTO DEL } \\
\text { DISPOSITIVO }\end{array}$} & $\%$ \\
\hline Conocen la capacidad de su dispositivo & $84 \%$ \\
\hline Tienen acceso a Redes Inalámbricas & $52 \%$ \\
\hline Tienen Bluetooth & $81 \%$ \\
\hline $\begin{array}{l}\text { Cuentan con tarjetas de almacenamiento } \\
\text { adicional. }\end{array}$ & $71 \%$ \\
\hline
\end{tabular}

Lo siguiente que nos interesaba conocer, era si sabían usar sus dispositivos para acceder a redes inalámbricas e intercambiar archivos vía Bluetooth, así como si podían descargar y visualizar diferentes tipos de formatos de archivo en sus dispositivos. Para ello se realizaron las siguientes preguntas:

10. Sabes conectarte a una red inalámbrica con tu dispositivo?

11.¿Cuál de los siguientes tipos de intercambio de archivos sabes hacer con tu dispositivo?

12. ¿Sabes descargar archivos de audio o video de Internet?

13. ¿Sabes conectar tu dispositivo a una computadora, con el cable USB?

14. ¿Sabes intercambiar archivos usando Bluetooth?. Responder aún si tu dispositivo no cuenta con Bluethoot

15.De la siguiente lista selecciona los tipos de archivos de datos que se pueden visualizar en tu dispositivo

\begin{tabular}{|l|l|}
\hline CONOCIMIENTO DE USO & $\mathbf{\%}$ \\
\hline Conectarse a una red inalámbrica & $66 \%$ \\
\hline Intercambiar audio & $84 \%$ \\
\hline Intercambiar video & $71 \%$ \\
\hline Intercambiar datos & $71 \%$ \\
\hline $\begin{array}{l}\text { Descargar archivos de audio o video de } \\
\text { Internet }\end{array}$ & $92 \%$ \\
\hline Conectar a otros medios & $99 \%$ \\
\hline Intercambiar archivos con Bluetooth & $93 \%$ \\
\hline Visualizar archivos Office y PDF & $75 \%$ \\
\hline
\end{tabular}

\section{PERCEPCIÓN E INTERES EN LA IMPLEMENTACIÓN DEL MODELO B-LEARNING}

Este bloque de preguntas, se plantearon con dos objetivos; por un lado, porque como cualquier producto o servicio, primero hay que analizar si existe la necesidad y la demanda, en este caso del b-learning en la Universidad, y por otro, como el modelo contempla usar el e-learning y las redes sociales, nos interesaba si tenían experiencia previa en estudios en línea y cuál de las redes sociales era la más utilizada. A continuación se exponen los resultados.

16. ¿Te gustaría poder estudiar en línea o a través de tu dispositivo móvil combinándolo con tus clases presenciales?

\begin{tabular}{|l|r|}
\hline $\begin{array}{l}\text { INTERÉS EN QUE SE IMPLEMENTE } \\
\text { EL MODELO B-LEARNING EN LA } \\
\text { UNIVERSIDAD }\end{array}$ & $\%$ \\
\hline $\mathrm{Si}$ & $99 \%$ \\
\hline $\mathrm{No}$ & $1 \%$ \\
\hline
\end{tabular}

17. En relación a la pregunta anterior. ¿Cuál de las siguientes razones consideras el principal beneficio de estudiar en esta modalidad, es decir, con material en línea en combinación con las clases presenciales? 


\begin{tabular}{|l|r|}
\hline BENEFICIOS DEL MODELO & \multicolumn{1}{|c|}{$\%$} \\
\hline Adquirir nuevos conocimientos & $42 \%$ \\
\hline Reforzar conocimientos & $55 \%$ \\
\hline Otros & $3 \%$ \\
\hline
\end{tabular}

18. ¿Has estudiado algún curso en modalidad virtual, conocida como e-learning?

\begin{tabular}{|l|r|}
\hline $\begin{array}{l}\text { CONOCIMIENTOS PREVIOS EN } \\
\text { E-LEARNING }\end{array}$ & \multicolumn{1}{l|}{$\%$} \\
\hline $\mathrm{Si}$ & $10 \%$ \\
\hline $\mathrm{No}$ & $90 \%$ \\
\hline
\end{tabular}

Este punto es preocupante, porque si los estudiantes terminan su carrera sin tener una experiencia previa en esta modalidad de enseñanza aprendizaje, seguramente se enfrentaran a problemas en el sector productivo, que como se menciono anteriormente, cada vez más frecuentemente utiliza esta modalidad para formar a sus colaboradores.

19. ¿En cuál de las siguientes redes sociales tienes una cuenta?

\begin{tabular}{|l|r|}
\hline PRINCIPALES REDES SOCIALES & \multicolumn{1}{|c|}{$\%$} \\
\hline Facebook & $34 \%$ \\
\hline MSN (Hotmail, myspace) & $41 \%$ \\
\hline Google & $16 \%$ \\
\hline Twitter & $9 \%$ \\
\hline
\end{tabular}

Solo se muestran las que obtuvieron mayor porcentaje.

\section{RESULTADOS DE LA ENCUESTA} APLICADA A LOS DOCENTES DE LA DIVISIÓN ECONÓMICO-ADMINISTRATIVAS DE LA UTCANCUN

La encuesta incluyo al $67 \%$ de la plantilla docente, 20 hombres y 13 mujeres, de los cuales el 100\% eran mayores de 40 años, 22 con grado de licenciatura, 10 con grado de Maestría y 1 con Doctorado. La encuesta estaba formada por 22 preguntas, 6 de información general del encuestado y 16 enfocadas a tres aspectos:
- Su familiarización con el modelo educativo por competencias de la Universidad.

- El conocimiento de los modelos de enseñanza virtuales.

- La disponibilidad, acceso y uso del medio

- La percepción e interés en la implementación del modelo b-learning

\section{SU FAMILIARIZACIÓN CON EL MODELO EDUCATIVO POR COMPE- TENCIAS DE LA UNIVERSIDAD}

En este apartado se incluyeron las siguientes preguntas:

1. ¿Está familiarizado con el modelo por competencias de nuestra Universidad?

2. ¿Aplica el modelo educativo por competencias?

3. $\mathrm{Su}$ conocimiento respecto a diseñar y aplicar rúbricas para evaluar competencias es:

4. Su conocimiento respecto al tema estilos de aprendizaje.

Este grupo de preguntas es muy importante, ya que permiten conocer el conocimiento en el modelo por competencias.

\begin{tabular}{|l|r|}
\hline CRITERIOS & \multicolumn{1}{c|}{$\%$} \\
\hline $\begin{array}{l}\text { Familiarización el modelo por } \\
\text { competencias }\end{array}$ & $97 \%$ \\
\hline Aplicación del modelo por competencias & $97 \%$ \\
\hline $\begin{array}{l}\text { Conocimiento para realizar rúbricas para } \\
\text { evaluar competencias. }\end{array}$ & $91 \%$ \\
\hline Conocimiento en estilos de aprendizaje & $100 \%$ \\
\hline
\end{tabular}

\section{CONOCIMIENTO DE LOS MODELOS DE ENSEÑANZA VIRTUALES}

Estas preguntas permiten conocer el conocimiento del docente sobre las modalidades de educación virtuales, los principales medios de conexión a redes y de trasferencia de archivos, así como su percepción sobre la importancia de innovación educativa. 
5. ¿Había usted escuchado sobre el concepto: m-learning?

6. ¿Había usted escuchado sobre el concepto: b-learning?

7. ¿Cuál de estos dos conceptos conoces?

8. ¿Considera usted importante la implementación de modelos de enseñanza aprendizaje innovadores en la formación de los estudiantes de la Universidad?

\begin{tabular}{|l|c|}
\hline \multicolumn{1}{|c|}{ CRITERIOS } & \multicolumn{1}{c|}{$\%$} \\
\hline Conocimiento sobre el m-learning? & $45 \%$ \\
\hline Conocimiento sobre el b-learning? & $70 \%$ \\
\hline Conocimiento del Bluetooth & $51 \%$ \\
\hline Conocimiento del wifi & $47 \%$ \\
\hline $\begin{array}{l}\text { Opinión sobre importancia de la } \\
\text { innovación tecnológica educativa. }\end{array}$ & $100 \%$ \\
\hline
\end{tabular}

\section{LA DISPONIBILIDAD, ACCESO Y USO DEL MEDIO}

9. De los siguientes dispositivos ¿cuál o cuáles tienes actualmente?

\begin{tabular}{|l|l|}
\hline DISPOSITIVO & RESP \\
\hline Celular o Smartphone & $56 \%$ \\
\hline Reproductor de Mp3 o Mp4 & $36 \%$ \\
\hline PDA & $8 \%$ \\
\hline
\end{tabular}

Las siguientes preguntas de este grupo, son importantes porque permiten identificar competencias específicas y técnicas, que se requieren en el modelo b-learning. Sin embargo, también de forma implícita nos proporcionan información sobre la actitud del docente hacia el modelo.

10.De las siguientes actividades, selecciona cuáles consideras que puedes realizar

11.Si tuvieras que crear un video de audio o video sobre un tema específico de la asignatura que imparte, cuánto tiempo consideras que debería durar.

12.¿Cuál de los siguientes materiales didácticos podrías elaborar como apoyo a tu materia?
13.De las siguientes opciones, seleccione cuál de ellas utiliza como herramientas de apoyo en la enseñanza de su materia.

\begin{tabular}{|l|l|}
\hline DISPOSITIVO & RESP \\
\hline $\begin{array}{l}\text { Intercambio de audio y video por } \\
\text { bluetooth }\end{array}$ & $29 \%$ \\
\hline Conexión entre dispositivos & $35 \%$ \\
\hline Intercambiar Tarjetas de almacenamiento & $12 \%$ \\
\hline $\begin{array}{l}\text { Subir archivos de audio y video a un sitio } \\
\text { web }\end{array}$ & $12 \%$ \\
\hline Crear sitios y administrar Redes sociales & $13 \%$ \\
\hline
\end{tabular}

\section{LA PERCEPCIÓN E INTERÉS EN LA IMPLEMENTACIÓN DEL MODELO B-LEARNING}

14. Te gustaría acceder a un sitio web en donde pudieras gestionar material didáctico de la materia que impartes y utilizarlos como complemento a tus clases presenciales?

\begin{tabular}{|l|c|}
\hline $\begin{array}{l}\text { INTERÉS EN QUE SE IMPLEMENTE } \\
\text { EL MODELO B-LEARNING EN LA } \\
\text { UNIVERSIDAD }\end{array}$ & $\%$ \\
\hline $\mathrm{Si}$ & $100 \%$ \\
\hline $\mathrm{No}$ & $0 \%$ \\
\hline
\end{tabular}

15.¿Cuál de las siguientes razones consideras el principal beneficio de tener una plataforma de este tipo?

\begin{tabular}{|l|l|}
\hline BENEFICIOS DEL MODELO & \multicolumn{1}{|c|}{$\%$} \\
\hline Mejorar mi práctica docente & $30 \%$ \\
\hline Aplicar mis conocimientos de las TIC & $14 \%$ \\
\hline $\begin{array}{l}\text { Usar varias herramientas Tecnológicas } \\
\text { en mis clases }\end{array}$ & $24 \%$ \\
\hline Dar material de reforzamiento & $32 \%$ \\
\hline
\end{tabular}

\section{CONCLUSIONES}

Esta investigación tiene su origen en el 2007, a raíz de un trabajo presentado en la maestría en TIC, sobre el m-learning y sus posibles aplicaciones en los procesos de enseñanza aprendizaje. Con estos ante- 
cedentes, la investigación aquí presentada, producto de una revisión bibliográfica y del estudio realizado con docentes y estudiantes en la División Económico Administrativa de la Universidad Tecnológica de Cancún, podemos decir que:

1.- El Modelo b-learning se ajusta más al modelo por competencias profesionales de la Universidad, que modalidades como el e-learning o el semipresencial.

2.- Como cualquier modelo o sistema, su implementación requiere de una visión estratégica por parte de los que toman las decisiones y asignan los recursos en las Universidades.

3.- Los docentes y estudiantes poseen los recursos, medios y el conocimiento para poder trabajar en el modelo b-learning.

4.- La aceptación e interés en el modelo blearning por parte de los docentes y estudiantes, no solo justifica su implementación, sino que lo exige.

5.- No existe mucha brecha entre los docentes y estudiantes en cuestión de conocimientos y usos de las TIC's, pero si las utilizan para diferentes fines y en distinta frecuencia.

6.- Los avances tecnológicos, continuamente están cambiado los roles de los docentes y estudiantes, pero aspectos como la constancia, dedicación y la disciplina, así como la colaboración y el desarrollo auto regulado siguen siendo las variables diferenciadoras.

\section{RECOMENDACIONES}

1. Proporcionar a los estudiantes de la Universidad, herramientas de formación, a través de modalidades mixtas de aprendizaje, con el fin de que al iniciar su vida laboral puedan ajustarse de manera efectiva y eficiente a los modelos de formación de las empresas, en donde cada vez es más frecuente la formación a distancia.

2.- Aprovechar que los docentes y estudiantes en su mayoría tiene acceso a Internet, cuentan con equipos de tecnología avanzada que les permite, administrar recursos como documentos, audio y video.

3.- Aprovechar los avances y logros que la Universidad ha tenido en la implementación del modelo de enseñanza aprendizaje por competencias profesionales y enfocar sus esfuerzos a la implementación del b-learning para que se complementen y se logren mejores resultados.

Finalmente, es preciso señalar que en el cuatrimestre enero-abril de 2011, en continuidad con esta investigación, se implemento el modelo b-learning, en dos grupos (53 alumnos) y se trabajo en forma tradicional en un grupo (23 alumnos). Lo anterior, con el fin de medir el impacto del modelo en los resultados académicos de los alumnos y comparar los resultados con el modelo tradicional.

\section{REFERENCIAS BIBLIOGRÁFICAS}

1. Bautista, G., Borges, F., Forés, A. (2006). Didáctica Universitaria en Entornos de Enseñanza-Aprendizaje Virtuales. Narcea Ediciones, Madrid-España.

2. Borges, F. (2007). El estudiante de entornos virtuales. Una primera aproximación. http://www.uoc.edu/digithum/9/dt/ esp/borges.pdf (visitado el 13-12-2010).

3. Cabero, J. (2006). Bases pedagógicas del e-learning. http://www.uoc.edu/ rusc/3/1/dt/esp/cabero.pdf (visitado el 17-11-2010).

4. Cabero, J. (2007). Las necesidades de las TIC en el ámbito educativo: oportunidades, riesgos y necesidades. http://investigacion. ilce.edu.mx/stx.asp?id=2281 (visitado el 15 de diciembre de 2010). 
5. Espíndola, J. (2000). Reingeniería Educativa. El Pensamiento Critico: cómo fomentarlo en los alumnos. 1ra. Ed., Pax México, D.F.-México.

6. Ferreiro, R. (2009). Estrategias didácticas del aprendizaje cooperativo. Métódo ELI. 2da. Ed., Editorial Trillas, D.F.-México.

7. Garcia, A. (2009). Experiencias de Innovación Docente Universitaria. 1ra Ed., Ediciones Universidad de Salamanca, Salamanca-España.

8. González, R. (2005). Nuevas Tecnologías Aplicadas a la Gestión de RRHH. Las TIC como herramienta de mejora permanente del Capital Humano. 1ra. Ed. Ideaspropias Editorial, Vigo. España.
9. Pacheco, L. (2007). La soledad del estudiante virtual caso de estudio personal. http://www.utn.edu.ar/aprobedutec07/ docs/264.pdf (visitado el 15 de diciembre de 2010).

10. Pereda, S. ; Berrocal, F. (2009). Gestión de Recursos humanos por Competencias. 2da. Ed., Editorial Universitaria Ramón Areces, Madrid-España.

11. Zulma, M. (2006). Aprendizaje autorregulado. Enseñar a aprender en diferentes entornos educativos. 1ra. Ed., Ediciones Novedades Educativas, Buenos AiresArgentina. 